\title{
Prolonged Toxicity after Amitriptyline Overdose in a Patient Deficient in CYP2D6 Activity
}

\author{
Jennifer Cohen Smith • Steven C. Curry
}

Published online: 26 May 2011

(C) American College of Medical Toxicology 2011

\begin{abstract}
Introduction Amitriptyline and its metabolite, nortriptyline, are metabolized, in part, by CYP2D6, a polymorphic enzyme. About $8 \%$ of Caucasians are deficient in CYP2D6 activity. Case Report We present the case of a comatose woman who intentionally overdosed on amitriptyline and displayed rising serum total tricyclic antidepressant concentrations for at least 6 days after admission. Serial immunoassay total tricyclic antidepressant concentrations in our patient showed gradual decline beginning day 7 , although the patient did not regain normal mental status until day 12 . Genotyping revealed the patient to be homozygous for the CYP2D6*4 allele, the most common explanation of CYP2D6 enzymatic deficiency among Caucasians. Patients taking tricyclic antidepressants who are homozygous for CYP2D6*4 demonstrate $>3$ times concentration-time curve (AUCs) and prolonged elimination half-lives, especially of secondary amines such as nortriptyline.

Discussion We believe this is the first report describing toxicokinetics after tricyclic antidepressant overdose in a CYP2D6-deficient patient.
\end{abstract}

Keywords Amitriptyline · Toxicokinetics ·

Pharmacokinetics · CYP2D6 - Overdose

J. C. Smith · S. C. Curry

Department of Medical Toxicology,

Banner Good Samaritan Medical Center,

Phoenix, AZ, USA

J. C. Smith $\cdot$ S. C. Curry

Department of Emergency Medicine, Maricopa Medical Center, Phoenix, AZ, USA

S. C. Curry $(\bowtie)$

Department of Medicine,

University of Arizona College of Medicine,

Phoenix, AZ, USA

e-mail: steven.curry@bannerhealth.com

\section{Introduction}

Overdose of amitriptyline and other tricyclic antidepressants is characterized, in part, by anticholinergic effects such as coma, respiratory depression and tachycardia, along with consequences of sodium channel blockade, including prolongation of the QRS complex and negative inotropy [1]. Despite anticholinergic action, which can slow down gut motility, patients usually exhibit onset of coma and lifethreatening cardiovascular events within a few hours of ingestion, and plasma drug concentrations usually peak within $24 \mathrm{~h}[2,3]$.

Amitriptyline and nortriptyline, its active metabolite, are metabolized by various cytochrome P450s, but especially by CYP2D6 and CYP2C19 [4, 5], with nortriptyline being mainly (>80\%) metabolized by CYP2D6 [6]. CYP2D6 is polymorphic, with about $8 \%$ of the Caucasian population demonstrating markedly slow or deficient activity [6]. We report the clinical course and total tricyclic antidepressant concentrations (amitriptyline+nortriptyline) in a patient who overdosed on amitriptyline. Sustained elevation of TCA concentration and prolonged coma led us to test for CYP2D6 genotypes associated with impaired metabolism, and a deficiency was identified. We believe this to be the first case describing the clinical course and serial drug concentrations in an identified CYP2D6-deficient patient who overdosed on a tricyclic antidepressant.

\section{Case Report}

A 55-year-old Caucasian woman was found unconscious and unresponsive with agonal respirations in her home by police after being alerted by friends. Several suicide notes were present that indicated the patient had taken 99 tablets of $25 \mathrm{mg}$ amitriptyline and 46 tablets of $1 \mathrm{mg}$ alprazolam. It was unclear what time the ingestion had taken place. At the 
scene, heart rate was 92 beats/min, blood pressure (BP) was $124 / 76 \mathrm{~mm} \mathrm{Hg}$, respiratory rate was 4 per minute (described as agonal), oxyhemoglobin fraction by pulse oximetry was $92 \%$ on room air, and fingerstick glucose concentration was $140 \mathrm{mg} / \mathrm{dl}$. She underwent endotracheal intubation and ventilation before transport to an outlying facility.

Upon arrival there, her heart rate was 106 beats $/ \mathrm{min}, \mathrm{BP}$ was $133 / 68 \mathrm{~mm} \mathrm{Hg}$, respirations were 12 per minute (assisted), and bladder temperature was $37.0^{\circ} \mathrm{C}$. Pupils were mid-position and reactive. The neurological examination was nonfocal and unremarkable except for coma. Funduscopic examination was normal. The chest was clear and heart tones were normal. Abdomen and extremities were unremarkable, and axillae were dry. Initial electrocardiogram (ECG) demonstrated sinus rhythm with QRS duration of $130 \mathrm{~ms}$. No gastrointestinal decontamination was performed. She was transferred to our institution.

Upon arrival at our facility, about $6 \mathrm{~h}$ after being found, she remained comatose and continued to require mechanical ventilation. Her examination was unchanged with heart rate 80 beats $/ \mathrm{min}$, BP $122 / 59 \mathrm{~mm} \mathrm{Hg}$, respirations 12 (assisted), and a temperature of $36.2^{\circ} \mathrm{C}$ (bladder). Serum electrolytes, glucose, liver function studies, and creatinine were normal. A complete blood count and prothrombin time were within normal limits. An ECG revealed normal sinus rhythm with a QRS of $148 \mathrm{~ms}$. She was given $100 \mathrm{mEq}$ of $\mathrm{NaHCO}_{3}$ (IV) and the QRS narrowed slightly to $138 \mathrm{~ms}$. Given the length of time after ingestion, we did not administer activated charcoal.

Later on the day of admission, approximately $12 \mathrm{~h}$ after being found, the patient developed hypotension unresponsive to fluid challenges, with systolic blood pressures transiently in the 70's mm Hg. Heart rate was 80 and she remained in sinus rhythm with a QRS duration of $124 \mathrm{~ms}$. Sodium bicarbonate $100 \mathrm{mEq}$ IV push resulted in a rise in systolic blood pressure into the $100 \mathrm{~s}$ and minimal narrowing of the QRS interval. She was commenced on a sodium bicarbonate infusion at $25 \mathrm{mEq} / \mathrm{h}$ that was continued overnight and discontinued the following morning. Blood pressure remained above $100 \mathrm{~mm} \mathrm{Hg}$ and stable, but the patient remained unresponsive without sedative administration.

Twelve and one-half hours after being found, a serum total tricyclic antidepressant level measured by immunoassay (Thermo Fisher Scientific, Fremont, CA) was 1,568 $\mu \mathrm{g} / \mathrm{l}$. A comprehensive urine drug screen that included gas chromatography/mass spectrometry showed only amitriptyline, nortriptyline, and cotinine. A urine immunoassay screen for benzodiazepines was positive, but the gas chromatography/mass spectrometry screen we use is incapable of detecting alprazolam, even when present, although it will detect several other benzodiazepines, which were absent. A specific urine drug screen for beta blockers and a serum lithium level were both negative.

On hospital days 3 and 4 she remained comatose, and serum total tricyclic antidepressant concentrations were 1,618 and 1,675 $\mu \mathrm{g} / 1$, respectively (Fig. 1). Six hours after the total serum tricyclic antidepressant concentration by immunoassay was $1,675 \mu \mathrm{g} / \mathrm{l}$, a blood sample was drawn and gas chromatography/mass spectrometry showed the plasma amitriptyline concentration to be $780 \mu \mathrm{g} / \mathrm{l}$ and nortriptyline $768 \mu \mathrm{g} / \mathrm{l}(\mathrm{total}=1,548 \mu \mathrm{g} / \mathrm{l})$. An MRI of the brain showed normal results.

By hospital day 6 , the patient was breathing spontaneously, opened her eyes, was making purposeful movements, and was following simple commands. Extubation was performed, but the patient was floridly anticholinergic with development of agitated delirium, picking behavior, and mumbling, nonsensical speech. She required endotracheal intubation once again, now for sedation and self-protection.

The peak serum total tricyclic antidepressant level was measured about 6.4 days after admission at $1,761 \mu \mathrm{g} / \mathrm{l}$, at which time the patient had heart rates in the $110 \mathrm{~s}$ and a QRS duration of $106 \mathrm{~ms}$. Total serum tricyclic antidepressant concentrations declined over the next several days (Fig. 1), and successful extubation was accomplished on hospital day 12, at which time her mental status was normal. She was discharged to a psychiatric facility on hospital day 14 .

On hospital day 6, blood was sent for CYP2D6 genotyping. Results returned showing she was homozygous for CYP2D6*4 alleles, a polymorphism associated with absent CYP2D6 activity [6]. We discussed results and implications with the patient and her private physician.

\section{Discussion}

In patients with large tricyclic antidepressant overdoses, significant neurological and cardiac toxicity generally occur

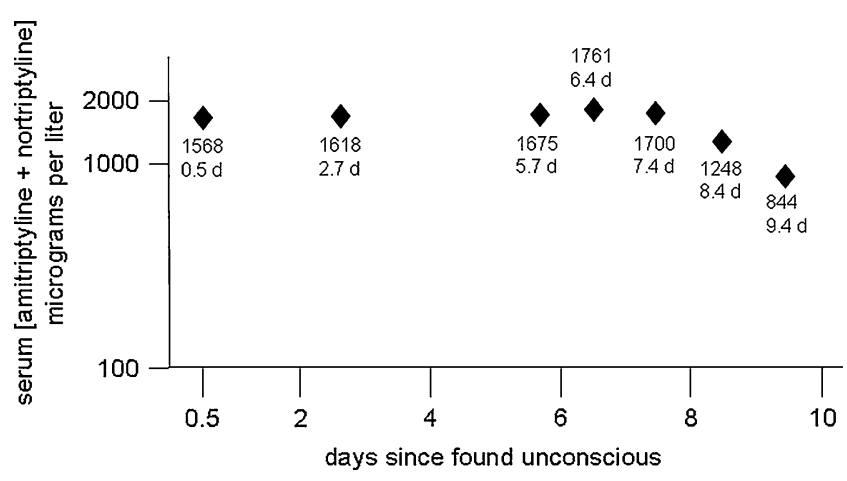

Fig. 1 Serum total amitriptyline and nortriptyline concentrations by immunoassay are shown for each data point in $\mu \mathrm{g} / \mathrm{L}$ (ddays) 
rapidly. Total serum tricyclic antidepressant levels peak within the first $24 \mathrm{~h}[2,3]$. CYP2D6 is partially responsible for metabolism of amitriptyline and responsible for more than $80 \%$ of nortriptyline's metabolism [4-6]. The most common allele responsible for reduced or absent CYP2D6 activity in $5-10 \%$ of Caucasians is CYP2D $6 * 4$, with homozygous persons commonly lacking CYP2D6 activity [7]. Amitriptyline displays between $30 \%$ and $60 \%$ bioavailability because of first-pass metabolism [8], and patients taking tricyclic antidepressants who are deficient in CYP2D6 demonstrate several times greater increases in the area under the concentration-time curve and prolonged elimination half-lives after therapeutic dosing compared to those with normal alleles $[6,9]$.

Pedersen and colleagues [10] described serum tricyclic antidepressant and maprotiline concentrations in 29 overdose patients and also described peak levels at the time of admission or within a relatively short time. Two of his patients maintained both amitriptyline and nortriptyline concentrations at relatively steady concentrations for 3 to 4 days before they began to decline. No metabolism status of CYP2D6 was reported.

Our patient experienced a prolonged rise in total drug concentration, and levels did not begin to decline until about 6.5 days of hospitalization. Deficiency of CYP2D6 most likely contributed to prolonged elevation of antidepressant levels in our patient. While nortriptyline toxicity has been described after therapeutic dosing in the face of CYP2D6 deficiency [11], we could not locate previous reports describing toxicokinetics after amitriptyline overdose in a CYP2D6-deficient patient.

Terminal elimination half-lives following overdose of tricyclic antidepressants in patients with unreported CYP2D6 metabolizing status have averaged $13-81 \mathrm{~h}[2$, 3, 9-11]. In our patient, the terminal elimination half-life of the total of amitriptyline and nortriptyline concentration was approximately $47.5 \mathrm{~h}$, based on the last three serum concentrations from days 7 through 9. Elimination half-life of secondary amines, such as desipramine, has been reported to be increased about three times in CYP2D6deficient metabolizers [9].

Alprazolam is mainly metabolized by CYP3A4 and exhibits an elimination half-life of nine to $16 \mathrm{~h}$. Alprazolam is neither a significant inhibitor of CYP2D6 nor CYP2C19. While alprazolam may have contributed to our patient's initial coma, she was clearly anticholinergic upon extubation on day 6, findings not expected based on alprazolam's pharmacologic effects and pharmacokinetics [12], but explained by documented persistently elevated antidepressant concentrations.

The serum total tricyclic immunoassay we used approximately quantifies tricyclic antidepressants and is calibrated using nortriptyline, with good cross-reactivity with amitrip- tyline. In our hands, total serum amitriptyline and nortriptyline concentrations are within $13 \%$ of those measured by chromatographic methods, and within-run variability and run-to-run variability coefficient of variations are both less than $1.7 \%$, in keeping with results of amitriptyline and nortriptyline concentrations measured by gas chromatography/mass spectrometry in our patient. Benzodiazepines and cotinine, found in the urine of our patient, do not cross-react with this assay. Substances such as quetiapine, that do cross-react with the assay, were notably absent by comprehensive screening of urine. The immunoassay we used served to confirm the delayed rise and prolonged elevation of tricyclic antidepressants and provided an estimation of terminal elimination half-life, but did not provide as an exact measurement of total amitriptyline and nortriptyline as would result from serial chromatographic measurements. The sustained and prolonged rise in drug levels along with clinical evidence of continued toxicity made us suspect CYP2D6 deficiency, which we confirmed with genetic testing.

\section{Conclusion}

Our patient, who was deficient in CYP2D6, experienced a delayed rise in total serum tricyclic antidepressant concentrations as well as prolonged intoxication.

\section{References}

1. Kerr GW, McGuffie AC, Wilkie S (2001) Tricyclic antidepressant overdose: a review. Emerg Med J 18(4):236-241

2. Franssen EJF, Kunst PWA, Bet PM et al (2003) Toxicokinetics of nortriptyline and amitriptyline: two case reports. Ther Drug Monit $25: 248-251$

3. Petit JM, Spiker DG, Ruwitch JF, Ziegler VE, Weiss AN, Biggs JT (1977) Tricyclic antidepressant plasma levels and adverse effects after overdose. Clin Pharmacol Ther 21:4751

4. Olesen OV, Linnet K (1997) Metabolism of the tricyclic antidepressant amitriptyline by cDNA-expressed human cytochrome P450 enzymes. Pharmacology 55(5):235-243

5. Olesen OV, Linnet K (1997) Hydroxylation and demethylation of the tricyclic antidepressant nortriptyline by cDNA-expressed human cytochrome P450 enzymes. Drug Metab Dispos 25 (6):740-744

6. Gardiner SJ, Begg EJ (2006) Pharmacogenetics, drugmetabolizing enzymes, and clinical practice. Pharmacol Rev 58:521-590

7. Neafsey P, Ginsberg G, Hattis D, Johns DO, Guyton KZ, Sonawane B (2009) Genetic polymorphism in cytochrome P450 2D6 (CYP2D6): population distribution of CYP2D6 activity. J Toxicol Environ Health B Crit Rev 12:362-388

8. Schulz P, Turner-Tamiyasu K, Smith G, Glacomini KM, Blaschke TF (1983) Amitriptyline disposition in young and elderly normal men. Clin Pharmacol Ther 33:360-366 
9. Koyama E, Sohn D-R, Shin S-G, Chiba K, Shin J-G, Kim Y-H et al (1994) Metabolic disposition of imipramine in oriental subjects: relation to metoprolol a-hydroxylation and $S$-mephenytoin 4 '-hydroxylation phenotypes. J Pharmacol Exp Ther 271:860-867

10. Pedersen OL, Gram LF, Kristensen CB, Moller M, Thayssen P et al (1982) Overdosage of antidepressants: clinical and pharmacokinetic aspects. Eur J Clin Pharmacol 23:513-521
11. Bertilsson L, Mellstrom B, Sjoqvist F, Martensson B, Asberg M (1981) Slow hydroxylation of nortriptyline and concomitant poor debrisoquin hydroxylation: clinical implications. Lancet 317:560561

12. Greenblatt DJ, Wright CE (1993) Clinical pharmacokinetics of alprazolam: therapeutic implications. Clin Pharmacokinet 24:453471 